\title{
Importancia de la hiperfosfatemia en la enfermedad renal crónica, cómo evitarla y tratarla por medidas nutricionales
}

\author{
Importance of hyperphosphatemia in chronic kidney disease, how to avoid it and treat it by nutritional \\ measures
}

\author{
Camila Andrea García Ospina ${ }^{1}$, María Cielo Holguín², Daniela Cáceres Escobar ${ }^{3}$, César Augusto Restrepo \\ Valencia ${ }^{4, *}$
}

${ }^{1}$ Estudiante de Enfermería, Universidad de Caldas, Manizales, Colombia

${ }^{2}$ Enfermera. Profesora Asociada, Universidad de Caldas, Manizales, Colombia

${ }^{3}$ Residente Medicina Interna, Universidad de Caldas, Manizales, Colombia

${ }^{4}$ Médico internista - nefrólogo. Profesor asociado, Universidad de Caldas y Universidad de Manizales, Manizales, Colombia

\begin{abstract}
Resumen
El manejo de los pacientes con enfermedad renal crónica (ERC) en todos los niveles de atención en salud es un reto frecuente y que amerita el conocimiento más apropiado y el mejor equipo de profesionales. El consumo de fósforo en la dieta y sus implicaciones sobre la función renal ha sido ampliamente descrito en la literatura, sin embargo, nuestros pacientes carecen de estrategias sencillas y de alto impacto sobre sus hábitos de alimentación, adaptadas a sus posibilidades económicas. Los objetivos de la presente revisión son, primero, proporcionar un conocimiento actualizado acerca de la hiperfosfatemia en la ERC y, segundo, compartir los resultados de una estrategia emprendida por un grupo multidisciplinario de profesionales para identificar el contenido de fósforo en los alimentos más consumidos por nuestros pacientes y adaptar una herramienta educativa para implementar en la consulta.
\end{abstract}

Palabras clave: insuficiencia renal cronica, hiperfosfatemia, aditivos alimentarios, etiquetado de alimentos, enfermedades cardiovasculares, hiperparatiroidismo.

http://doi.org/10.22265/acnef.4.1.270

\begin{abstract}
Treatment of patients with chronic kidney disease (CKD) at all levels of healthcare, is a frequent challenge that deserves the best expertise and the best professional team. Phosphorus ingest in the diet and its implications for kidney function have been widely depicted in the literature. However, our patients lack of simple strategies that have a high impact on their eating habits within their economical possibilities. The goals of the present review are to provide a multidisciplinary group of professionals with an updated insight into hyperphosphataemia in CKD and share the results of a strategy carried out by a group of cross-disciplinary professionals, to identify the amount of phosphorus found in the most consumed foods by our patients, and to adapt of a new educational tool in order to be implemented during consultation.
\end{abstract}

Key words: Renal insufficiency, chronic, hyperphosphatemia, food additives, food labeling, cardiovascular disease, hyperparathyroidism.

http://doi.org/10.22265/acnef.4.1.270

Referenciar este artículo: García Ospina CA, Holguín Cielo M, Cáceres Escobar D, Restrepo Valencia CA. Importancia de la hiperfosfatemia en la enfermedad renal crónica, cómo evitarla y tratarla por medidas nutricionales. Rev. Colomb. Nefrol. 2017;4(1): 38 - 56.

Correspondencia: *César Augusto Restrepo Valencia, caugustorv@une.net.co

Recibido: 30-11-16 • Aceptado: 07-02-17 • Publicado en línea: 10-03-17 


\section{Introducción}

$\mathrm{E}$ n el último siglo, las sociedades modernas han cambiado de manera dramática sus hábitos alimentarios, cambios para los que, sin duda, no estábamos preparados. Para la población de pacientes con enfermedad renal crónica (ERC) el panorama se hace mucho más complejo si consideramos la dieta como pilar importante en el control de la enfermedad y la prevención de las complicaciones en todos sus estadios evolutivos. En concordancia con lo anterior, debemos contar con un conocimiento actualizado que nos permita proporcionar la mejor educación y las medidas necesarias a este grupo de pacientes y así impedir que continúen llevando enemigos en su cesta de mercado. En el presente documento nuestro propósito fue, en primera instancia, realizar una revisión actualizada sobre las implicaciones del fósforo en la salud del enfermo renal y adicionalmente, por medio de un trabajo de campo, identificar su presencia en los alimentos más consumidos en nuestro medio que se destacan por su alto contenido en fosfatos. Con ello, nuestro grupo propone la creación de herramientas más útiles y cercanas que puedan lograr mejores resultados en la salud de estos pacientes incluso desde estadíos tempranos de la enfermedad.

\section{Fósforo y enfermedad renal}

El descubrimiento del fósforo data de 1669, cuando el alquimista alemán Henning Brandt logró aislarlo a partir de grandes cantidades de orina como un polvo blanco que brillaba en la oscuridad. La palabra fósforo, según su etimología, significa "luz brillante", y proviene del latín phosphorus que se originó a su vez del griego phosphoros, formado por phos, luz, y phoros, portador: "portador de luz"1. Es el segundo mineral más abundante en el organismo, representa aproximadamente el $1 \%$ del peso corporal (600-800 g), y, en cuanto a su distribución, $85 \%$ se encuentra en el esqueleto, 14 $\%$ está disponible a nivel celular y solo $1 \%$ en el espacio extracelular ${ }^{2}$. Se conoce bien la importante función que cumplen los riñones al mantener la adecuada excreción de fósforo en la orina y de esta manera lograr que sus niveles séricos sean los adecuados para el cumplimiento de diversas funciones. El estrecho equilibrio entre ingesta y eliminacion renal da lugar a que incipientes cambios en las concentraciones de este ion indiquen compromiso de la función renal, lo que nos permite de forma oportuna intervenir los pacientes, procurando detener el ominoso curso hacia complicaciones bien reconocidas como el hiperparatiroidismo secundario (HPTS). Desde hace tiempo, los científicos se han inquietado por reconocer las mejores estrategias para evitar la elevación de sus niveles séricos. Dentro de ellas, con justa razón la dieta ha sido uno de los campos de estudio más relevantes. Daremos un repaso por la fisiología del metabolismo del fósforo y de esta forma nos adentraremos de manera más ilustrada en las discusiones sobre este tema.

Al ser el fósforo uno de los minerales más abundantes del cuerpo humano, se relaciona con funciones tan importantes como la estructura de las células (síntesis del ADN, lípidos de membrana y señalización intracelular), metabolismo celular (generación de ATP), mantenimiento de homeostasis ácido-base y mineralización ósea ${ }^{3}$. Los valores de referencia en el plasma de adultos se encuentran en el rango de 2.8 a $4.5 \mathrm{mg} / \mathrm{dl}^{2}$. La ingesta diaria de fósforo por vía oral en forma de fosfato $\left(\mathrm{PO}_{4}\right)$ varía entre 700 y $2000 \mathrm{mg}$, de acuerdo a la proporción de alimentos ingeridos ricos en este mineral. Una vez está disponible para su metabolismo, puede ser utilizado a nivel celular u optar por depositarse en la matriz ósea o tejidos blandos. Su excreción puede ser renal en un valor entre 600 y $1500 \mathrm{mg} /$ día, o por tubo digestivo en menor valor cercano a $500 \mathrm{mg}^{4}$. La dieta occidental es rica en $\mathrm{PO}_{4}$ y los alimentos que se destacan por su alto contenido incluyen carne roja, pescados, lácteos y aditivos alimentarios. Al ser ingeridos estos alimentos, el $\mathrm{PO}_{4}$ es absorbido a nivel del intestino delgado, actividad favorecida por la forma activa de la vitamina D (1,25 dihidroxi 
vitamina D), que estimula la actividad de la proteína NaPillb ubicada en el borde en cepillo de la membrana luminal. Es importante anotar que este proceso no es facilmente saturable y que, a mayor consumo de fosfatos, mayor absorción ${ }^{5}$. Por otro lado, los riñones cumplen un papel primordial en la homeostasis del fósforo, debido a su capacidad para reabsorber el fosfato de la orina, actividad fuertemente influenciada por los aportes de fosfatos en la dieta. Un adecuado balance en estos dos sistemas puede lograrse gracias a un especializado conjunto de procesos, en los que intervienen la vitamina $\mathrm{D}$, el calcio, la paratohormona $(\mathrm{PTH})$ y las fosfatoninas, entre otros. A nivel renal, el control de la reabsorción de fosfatos se regula por dichos factores hormonales y metabólicos ${ }^{4}$ (Tabla 1), modificándose la actividad de los cotransportadores a nivel del túbulo próximal en cuestión de minutos, horas o días dependiento del tipo de co-transportador ${ }^{6,7}$.

Tabla 1. Factores que alteran la regulación renal de fosfato

\begin{tabular}{|l|l|}
\hline Incremento de la absorción renal del fosfato & Disminución de la absorción renal \\
\hline 1,25 hidroxi vitamina d & PTH \\
\hline \multirow{5}{*}{ Hormona Tiroidea } & Fosfatoninas (Ejemplo: FGF23) \\
\hline & Dieta rica en fosfatos \\
\cline { 2 - 2 } & Acidosis metabólica \\
\cline { 2 - 2 } & Deficiencia de potasio \\
\hline & Glucocorticoides \\
\hline & Dopamina \\
\hline & Hipertensión \\
\cline { 2 - 2 } & Estrógenos \\
\hline
\end{tabular}

FGF23: Factor de crecimiento fibroblástico 23

Tomado y adaptado de la referencia 4 (Clin J Am Soc Nephrol 2015;10:1257-1272).

Dentro de los factores hormonales que se deben destacar en la enfermedad renal, el Factor de crecimiento fibroblástico 23 (FGF23) y la PTH cumplen una función relevante, que se inicia a partir de la instauración del deterioro de la función renal cuando el fósforo incrementa sus niveles en el organismo. La diminución de la tasa de filtración glomerular (TFG) aumenta los niveles de fósforo séricos, lo que estimula la producción de FGF23 por los osteocitos, éste ejerce su función gracias a la presencia del cofactor Klotho producido en el riñón. Este permite activar el receptor FGF1 y de esta manera disminuir la actividad de las proteínas transportadoras NaPiIIa y NaPiIIc en las células del túbulo contorneado proximal. De manera simultánea, posibilita disminuir la actividad de la 1-hidroxilasa, y aumentar la de la 24-hidroxilasa, permitiendo la disminución de los niveles circulantes de $1,25 \mathrm{OH}$ vitamina $\mathrm{D}$, lo que lleva finalmente a una disminución en la absorción intestinal de fósforo y calcio, restaurándose la normofosfatemia. Sin embargo, la caída en los niveles de calcio sérico estimula la secreción de PTH, que contribuye a estimular la fosfaturia al también inhibir la actividad de las proteínas NaPiIIa y NaPiIIc. La PTH, a diferencia del FGF23, aumenta la actividad de la 1-hidroxilasa renal, promoviendo la síntesis de la vitamina $\mathrm{D}$ activa y estimulando la actividad osteoclástica con mayor reabsorción ósea y restauración del calcio sérico ${ }^{4,8,9}$. 
De este modo, mediante las dos vías mencionadas se logra un efectivo mecanismo reductor de los niveles séricos de fosfatos. La ingesta dietaria se contrabalancea con un aumento en la excreción por unidad nefronal, lográndose un balance neutral de fosfatos ${ }^{4}$. Sin embargo, los mecanismos de compensación descritos son temporales y la prolongada exposición a una sobrecarga oral de fosfatos asociada a reducción en la masa nefronal lleva a un exceso de trabajo tubular y a la generación de lesiones tubulointersticiales. La hiperfosfatemia refleja desajustes en el metabolismo del fosfato por falla renal descompensada entre la absorción intestinal y excreción urinaria, lo que ocurre cuando la TFG se acerca a un valor de $30 \mathrm{ml} / \mathrm{min}$. Lo anterior refleja la importancia de una dieta adecuada para evitar la activación de la totalidad de los mecanismos compensadores expuestos ${ }^{10}$.

Después de este breve repaso sobre los procesos que se llevan a cabo y los agentes que intervienen en la homeostasis del fósforo, podemos comprender cómo su consumo en la dieta es un regulador directo de su propia reabsorción a nivel renal, ya que logra protagonismo en el curso de la ERC debido al desarrollo de desórdenes óseos y minerales; eventos cardiovasculares; y afectación en la supervivencia tras años de evolución. Esta problemática se representa bien en las altas tasas de mortalidad, que superan el $20 \%$ a pesar de la introducción de la terapia dialítica en la segunda mitad del siglo $\mathrm{XX}^{11}$. Desde entonces, la historia natural de la enfermedad renal se ha reescrito para traer más retos y nuevas oportunidades de intervención.

Ahora daremos paso a las discusiones y exposición de la evidencia sobre ERC y consumo de fósforo, con el propósito inicial de dar respuesta a los interrogantes más frecuentes de quienes se enfrentan en su práctica diaria al reto de lograr el equilibrio más apropiado para estos pacientes. A su vez, pretendemos proponer herramientas que sean de fácil aplicación, eficaces y con impacto favorable en el control de la enfermedad.

\section{¿Cómo interpretar los niveles séricos de fósforo en el paciente con ERC?}

Los niveles séricos de los que disponemos a diario son un reflejo de la concentración de fósforo inórganico plasmático, medida por la mayoría de laboratorios en $\mathrm{mg} / \mathrm{dl}$. A pesar de que este representa una ínfima parte del fósforo total del cuerpo, en este compartimento está representado el consumo dietario agregado cada día; desde allí se realiza el intercambio con los tejidos y el fósforo órganico. Según lo anterior, podemos entender, por una parte, por qué la medición de fósforo intracelular (células de sangre periférica) no se considera práctica en el escenario clínico habitual, y, por la otra, cómo la intención de predecir la ingesta con la medición habitual es bastante limitada. En el paciente con ERC, está suficientemente establecida la relación entre el consumo dietario de fósforo y los niveles séricos, no siendo así para la población de pacientes no renales. La medición del fósforo urinario es una herramienta adicional para proporcionar información sobre la ingesta y absorción intestinal ${ }^{12}$. Se debe diagnosticar hiperfosfatemia cuando se presenten niveles séricos por encima de $4,5 \mathrm{mg} / \mathrm{dl}$.

\section{¿Puede el consumo de fósforo llevar al desarrollo de enfermedad renal aguda?}

Múltiples publicaciones han logrado establecer un claro riesgo de desarrollo de nefropatía aguda por fosfatos tras la exposición a soluciones orales de fosfato sódico, con daño renal en ocasiones irreversible. Estos estudios han permitido identificar factores de riesgo para el establecimiento de dicha lesión: sexo femenino, edad avanzada, hipertensión arterial y enfermedad renal establecida ${ }^{13}$. Los hallazgos histológicos característicos de esta lesión son reconocidos por depósito de cristales de fosfato de calcio en las células y lúmenes epiteliales tubulares; inflamación intersticial; fibrosis; y apoptosis de las células tubulares ${ }^{14}$. El riesgo en pacientes no renales parece ser claramente inferior. Un seguimiento de la función renal en población sana expuesta a preparación para colonoscopias no ha sido asociado con injuria renal y pone de manifiesto la importancia de la hidratación durante la fase de preparación ${ }^{15}$. 


\section{Fósforo y progresión de la ERC}

En las etapas iniciales de la enfermedad renal los pacientes logran mantener el equilibrio, permaneciendo con niveles normales de fósforo. Una vez que la TFG continúa su descenso, la imposibilidad de una adecuada excreción de fósforo se convierte en el sustrato fisiopatológico para el desarrollo de desórdenes minerales y óseos. No obstante, el impacto de la hiperfosfatemia en la comorbilidad del paciente renal tiene otras importantes implicaciones; por ello, gran parte de los esfuerzos que se le han dedicado hasta la actualidad se han centrado en reconocer tanto su asociación con la progresión de la enfermedad renal, como otros factores asociados y la influencia de la carga de fósforo independiente de los niveles séricos. Esto se intentó demostrar en un estudio español publicado en 2011, que describe cómo la función renal (en mayor grado), el sexo femenino, la albúmina sérica, el bicarbonato sérico y el uso de diuréticos resultaron ser determinantes significativos de los niveles de fósforo sérico. En este mismo estudio, se logró demostrar que la relación del fósforo sérico con la velocidad de deterioro de la función renal es más intensa cuanto más alta es la función renal residual basal, lo que sugiere el papel independiente del fósforo, o de los factores relacionados, con las concentraciones de fósforo ${ }^{16}$. En el pasado se ha descrito, si bien a través de un menor número de publicaciones, cómo el fósforo es un fuerte predictor independiente de disminución de la función renal en pacientes con ERC en estadios avanzados ${ }^{17}$. Por el sustento que brindan los hallazgos mencionados, se ha logrado establecer dicha relación en pacientes desde estadio 3 . Un estudio observacional que incluyó 225 pacientes con enfermedad renal con una media de TFG de 43 +/- 19 $\mathrm{ml} /$ min pudo demostrar que el fósforo sérico basal predice la progresión de la disfunción renal durante un seguimiento de 2,5 años, previo ajuste de los factores conocidos de progresión de daño renal tales como edad, proteinuria y presión arterial sistólica $(\mathrm{PAS})^{18}$. De esta manera, la evidencia disponible nos demuestra que la asociación entre los niveles de fósforo y la velocidad de la progresión renal es explicada por la gravedad de la enfermedad renal.

\section{¿Es la hiperfosfatemia un factor de riesgo cardiovascular independiente en los pacientes con ERC?}

Hasta ahora se ha demostrado que los factores de riesgo cardiovascular clásicos como la obesidad, hiperlipidemia, diabetes mellitus, hipertensión y tabaquismo no logran explicar satisfactoriamente el mayor riesgo cardiovascular en los pacientes con ERC desde las etapas tempranas de la enfermedad. De este modo se ha logrado identificar que los niveles elevados de fósforo sérico tienen un papel central en la inducción de calcificación vascular. Un estudio clásico y de consulta obligada en este tema, publicado en los años setenta, describió, en la autopsia realizada a pacientes que se encontraban en estadios avanzados de la enfermedad renal con o sin terapia dialítica, que la calcificación de la media de los vasos estaba presente en el $79 \%$ del grupo de pacientes en diálisis, en comparación con el $44 \%$ del grupo sin dicho tratamiento y con mayor compromiso en los vasos de corazón, pulmones, estómago y riñones ${ }^{19}$. Estudios observacionales de individuos con y sin enfermedad renal clínicamente manifiesta también demuestran que los mayores niveles de fósforo sérico se asocian con enfermedad cardiovascular manifiesta y calcificación vascu$1 \mathrm{lar}^{20-23}$. Otro estudio concluye que las concentraciones más altas de fosfato en suero, aunque todavía dentro del rango normal, se asociaron con una mayor prevalencia de calcificación vascular y valvular en las personas con ERC moderada ${ }^{24}$, lo que lo que da elementos de peso para continuar la discusión acerca de la influencia de la carga de fósforo y progresión de la ERC, independientemente de los valores de fósforo sérico. Otros hallazgos sobre los efectos del fósforo lo relacionan con aumento de la masa ventricular izquierda en pacientes con ERC, siendo un hallazgo en estudios el predominio en población femenina ${ }^{25,26}$ 
¿El control del fósforo puede retrasar la progresión de la ERC o mejorar el pronóstico?

Es universalmente reconocido que los pacientes con ERC se enfrentan a elevadas tasas de mortalidad, por ello la viva necesidad en la comunidad científica de identificar los factores de riesgo modificables que impacten en la historia natural de la enfermedad y la calidad de vida. Niveles de fósforo $>$ de $5.0 \mathrm{mg} / \mathrm{dl}$ se han asociado con mayor riesgo relativo de muerte en una población de pacientes en hemodiálisis ${ }^{11}$. Estos hallazgos son reproducibles en estudios que evaluaron este aspecto en pacientes con ERC sin terapia dialítica. Una publicación aparecida en 2005, que incluyó una población de 6730 veteranos durante el período de 1999 a 2002, observó una asociación entre los niveles séricos elevados de fósforo y el riesgo de mortalidad e infarto agudo de miocardio (IAM) entre los pacientes con ERC, independientemente de la función renal y otros factores de confusión conocidos, con un HR para muerte de 1,9 en los individuos con fósforo $>5 \mathrm{mg} /$ $\mathrm{dl}^{27}$. De los resultados obtenidos en este estudio, llama especialmente la atención que el riesgo se haya incrementado a partir de un nivel sérico considerado dentro del límite superior de normalidad $(>4.5$ $\mathrm{mg} / \mathrm{dl})$.

Con lo anterior queda claramente el modo cómo deberían fundamentarse estrategias para la disminución del fósforo sérico en los pacientes con ERC. Sin embargo, no resulta adecuado limitar los esfuerzos terapéuticos a iniciar quelantes del fósforo en los pacientes que desarrollen hiperfosfatemia. Se ha posicionado un nuevo paradigma frente a la restricción del fósforo, que nos plantea tomar medidas independientemente de los niveles reportados en sangre. Siendo el escenario ideal para iniciar la intervención el momento en que los niveles de FGF23 comiencen a elevarse (a partir del estadio 2 de ERC), debido a que este es el signo de exceso de ingesta de fosfato en relación con el número de nefronas ${ }^{28}$. En este punto se hacen necesarias medidas tempranas que puedan prevenir el daño tubular y, por lo tanto, retrasar la progresión de la $\mathrm{ERC}^{29}$. En pacientes con enfermedad renal temprana y avanzada sometidos a dietas bajas en proteína, se ha logrado obtener ni- veles de FGF23 más bajos ${ }^{30}$. Se plantea, entonces, la pertinencia de realizar seguimiento con niveles séricos de FGF23 y no con fósforo sérico, pues este, como ya se mencionó, puede permanecer dentro de rangos de normalidad y podría ser una explicación acertada al interrogante de por qué estudios con grandes cohortes de pacientes con ERC en estadios tempranos no han podido demostrar una asociación independiente del fósforo sérico con el riesgo de muerte y progresión de la enfermedad renal ${ }^{31}$.

\section{Fósforo y dieta}

Una especial atención merece la relación que se tiene entre la ingesta diaria de fósforo y su contribución en los deslenlaces del paciente con diagnóstico de ERC. La evaluación de la ingesta de fósforo en la dieta es un reto para el clínico debido tanto a las limitaciones para su cuantificación en los alimentos como al creciente uso de aditivos fuente de fósforo inorgánico a nivel mundial.

Los fosfatos en la dieta aparecen bajo dos formas: fósforo orgánico e inorgánico.

Fósforo orgánico: se encuentra en los alimentos ricos en proteínas, tanto de origen animal como vegetal. El fósforo orgánico es hidrolizado en el tracto intestinal y posteriormente se absorbe como fósforo inorgánico. Solo del 30 al 60 \% del fósforo orgánico es absorbido y su fuente principal son las carnes de mamíferos, aves, pescados, huevos y lácteos. El fósforo de la proteína de origen animal se encuentra principalmente en el compartimento intracelular de los alimentos, pero es de mayor digestibilidad que el fósforo de la proteína vegetal.

El fósforo orgánico de origen vegetal es abundante en semillas, frutos secos y legumbres ${ }^{32}$. A diferencia del fósforo de la proteína de origen animal, el de la proteína vegetal se encuentra incorporado al ácido fítico o fitato, un ácido orgánico cuya principal función es almacenar fósforo como fuente de energía para la planta. El ácido fítico establece fuertes uniones iónicas con minerales esenciales en la nutrición, formando quelatos insolubles que no pueden ser absorbidos por el organismo. 
El ser humano y los animales no rumiantes presentan limitada capacidad para hidrolizar el ácido fítico, debido a que carecen de la enzima fitasa, necesaria para separar el fósforo del fitato. Por ello, la biodisponibilidad del fósforo de la proteína vegetal es relativamente baja, usualmente menor del $50 \%$.

En pacientes sin ERC, si se les administra la misma cantidad de fósforo proveniente de alimentos animales y de vegetales, la proteína de origen vegetal no cubrirá el requerimiento proteico, pero ayudará a un mejor manejo del fósforo, aunque podría resultar en un inadecuado aporte proteico.

Fósforo inorgánico: se utiliza principalmente como aditivo en los alimentos. En ellos tienen diversas aplicaciones y funciones: reguladores del $\mathrm{pH}$, antioxidantes, estabilizantes proteicos, potenciadores del sabor, colorantes, sales en quesos, mejoradores de masas y levaduras químicas, emulsionantes, humectantes, leudantes, secuestrantes y espesantes.

Son fuentes comunes de fósforo inorgánico las bebidas, comidas congeladas, cereales para el desayuno, quesos procesados y para untar, productos instantáneos, comidas precocinadas, salchichas, galletas y otros ${ }^{33}$.

El fósforo inorgánico no está unido a las proteínas, sino que se presenta en forma de sales (polifosfatos, ácido fosfórico) rápidamente disociables, por lo que fácilmente se absorben en el tracto intestinal. Entre el 90 y el $100 \%$ del fósforo inorgánico se absorbe a nivel intestinal, a diferencia del 40-60\% del fósforo orgánico presente en los alimentos ${ }^{34-37}$.

Infortunadamente, las empresas productoras de alimentos no están obligadas a presentar el contenido de fósforo en las etiquetas alimentarias. Para la nutricionista representa un problema el cálculo de la ingesta real de fosforo por los pacientes, puesto que hoy en día, y por comodidad, para toda la población en general el acceso a los alimentos procesados va en aumento, al igual que el consumo de fósforo inorgánico.
Nuestro grupo de trabajo se planteó la necesidad de calcular el fósforo total de los alimentos y de esta manera diseñar dietas más acertadas para los pacientes desde estadios tempranos de la enfermedad. Para ello se diseñó un trabajo de campo, con el fin de documentar el contenido de fósforo en los alimentos previamente reconocidos por su alto contenido de fosfatos en tiendas y supermercados de la ciudad de Manizales. Para nuestra sorpresa, la cantidad de fósforo añadido (en miligramos o gramos) no se encontró etiquetada en la gran mayoría de los productos seleccionados. Esto nos condujo a la búsqueda de la normativa actual, en la que encontramos que los fabricantes no se encuentran en obligación de ofrecer una información de tanta importancia. Adicionalmente, los aditivos fosfóricos que son incluidos en la lista de ingredientes pasan facilmente desapercibidos para los consumidores debido a los múltiples nombres utilizados. En la Tabla 2 se mencionan algunos de ellos con su respectiva codificación según la normativa establecida en la Union Europea.

\begin{tabular}{|c|c|}
\hline \multicolumn{2}{|c|}{ Tabla 2. } \\
\hline Aditivos fosfóricos. Los más importantes: fosfatos y derivados \\
Aditivos fosfóricos \\
Ácido fosfórico & Representación \\
\hline $\begin{array}{c}\text { Ortofosfatos (Fosfato sodio, } \\
\text { potasico, calcico y amonio) }\end{array}$ & E339-E340-E341-E342-E343 \\
\hline Difosfatos & E450 \\
\hline $\begin{array}{c}\text { Trifosfatos (pentasódico y } \\
\text { pentapotásico) }\end{array}$ & E451 \\
\hline $\begin{array}{c}\text { Polifosfatos (de sodio, potasio, } \\
\text { calcio y amonio) }\end{array}$ & E452 \\
\hline Fosfátidos de amonio & E442 \\
\hline Fosfato ácido de sodio y aluminio & E541 \\
\hline $\begin{array}{c}\text { Potenciadores del sabor: ácido } \\
\text { guanílico (Monofosfato de } \\
\text { Guanosina) }\end{array}$ & E626-E629 \\
\hline $\begin{array}{c}\text { Potenciadores del sabor: ácido } \\
\text { inosínico (inosianato) }\end{array}$ & E630-E633 \\
\hline
\end{tabular}

Fuente: REGLAMENTO (UE) No 1129/2011 DE LA COMISIÓN de 11 de noviembre de 2011 por el que se modifica el anexo II del Reglamento (CE) número 1333/2008 del Parlamento Europeo y del Consejo para establecer una lista de aditivos alimentarios de la Unión ${ }^{38}$ 
Para esta Comunidad, en todos los idiomas que la conforman, la letra $\mathrm{E}$ identifica un aditivo, entendiéndose como aditivo alimentario a aquella sustancia que, sin constituir por sí misma un alimento ni poseer valor nutritivo, se agrega intencionalmente a los alimentos y bebidas en cantidades mínimas con objetivo de modificar sus caracteres organolépticos o facilitar o mejorar su proceso de elaboración o conservación ${ }^{38}$.
En nuestro estudio utilizamos esta codificación para nombrar los aditivos encontrados en los productos, elegidos por su consumo frecuente en nuestra dieta y por ser reconocidos como de alto contenido en fósforo (Tabla 3). En esta última tabla se puede observar que el aditivo está indicado en todos los productos sin indicar su gramaje y solo para los granos se encontró una cifra numérica (Figura 1).

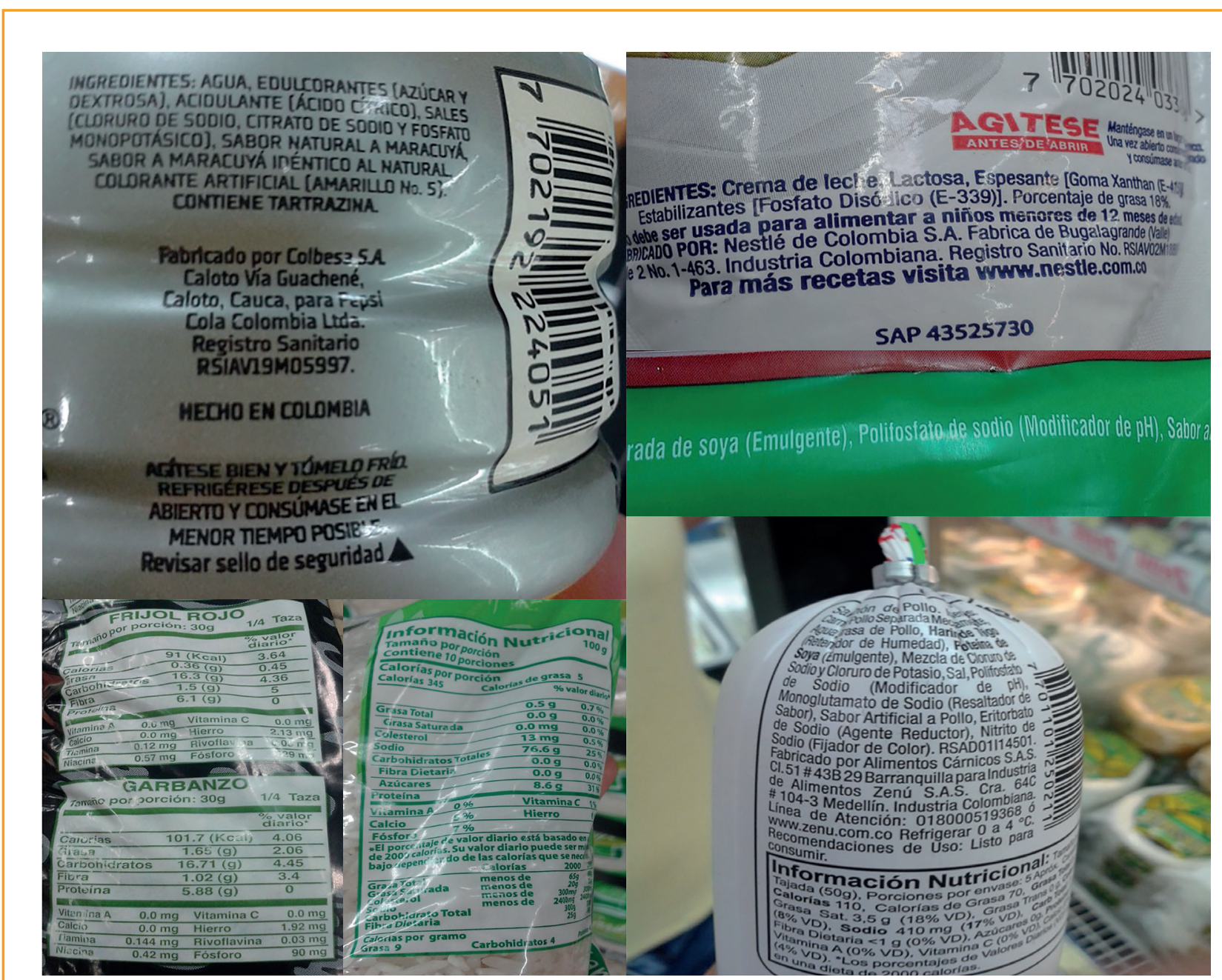

Figura 1. Formas de presentación de fósforo en diversos alimentos 


\begin{tabular}{|c|c|c|c|}
\hline \multicolumn{4}{|c|}{ Presencia de aditivos fosfóricos en alimentos básicos de la canasta familiar } \\
\hline Grupo de alimentos & Proteína (g) & Fósforo (mg)- aditivos & Sodio (mg) \\
\hline \multicolumn{4}{|l|}{ Lácteos y derivados } \\
\hline Leche entera Éxito & 6 & E452 & 130 \\
\hline Leche entera Celema & 6 & E451 & 100 \\
\hline Leche semideslactosada Éxito & 6 & E452 & 150 \\
\hline Leche semidescremada Colanta & 6 & $\mathrm{E} 452$ & 110 \\
\hline Leche & semidescremada & semideslactosada & \\
\hline Alpina & 6 & E451 & 85 \\
\hline Leche $0 \%$ grasa Alquería & 6 & E452 & 90 \\
\hline Leche de soya integral Toning & 13 & $6.6-\mathrm{E} 339$ & 170 \\
\hline Leche en polvo El Rodeo & 5 & E339 & 70 \\
\hline Queso semiduro graso Colanta & 6 & E451 & 220 \\
\hline Queso mozarella Alpina & 7 & E451 & 180 \\
\hline Queso Finesse Alpina & 7 & E451 & 220 \\
\hline Queso campesino Colanta & 6 & E451 & 120 \\
\hline Queso Lonchi semigraso & 5 & E451 & 85 \\
\hline \multicolumn{4}{|l|}{ Carnes embutidas } \\
\hline Chorizo santarrosano precocido & 10 & E451 & 560 \\
\hline Chorizo de cerdo Ranchera & 5 & E452 & 410 \\
\hline Chorizo campesino Colanta & 8 & E451 & 560 \\
\hline Chorizo Olímpica & 7 & $\mathrm{E} 452$ & 430 \\
\hline Mortadela $96 \%$ libre de grasa Rica & 5 & $\mathrm{E} 452$ & ----- \\
\hline Mortadela Zenú & 5 & E452 & \\
\hline Mortadela de pollo Zenú & 5 & E452 & 350 \\
\hline Costilla de cerdo ahumadas Ranchera & 16 & E452 & 260 \\
\hline Jamón Pietrán & 6 & E452 & 130 \\
\hline Jamón Premium Pietran & 7 & $\mathrm{E} 452$ & 350 \\
\hline Salchichón de Res & 7 & $\mathrm{E} 452$ & 440 \\
\hline Salchichón de pollo Zenú & 6 & E452 & 410 \\
\hline Salchichas Rica & 4 & $\mathrm{E} 452$ & 440 \\
\hline Salchichas Ranchera & 4 & E452 & 410 \\
\hline Salchicha enlatada Zenú & 8 & $\mathrm{E} 452$ & 450 \\
\hline Salchicha de pavo & 10 & E451 & 350 \\
\hline Salchichas Viena Especial & 2 & E340 & 270 \\
\hline Salchicha Llanera & 4 & E452 & 320 \\
\hline Rollo de carne de res Rica & 10 & E452 & 650 \\
\hline Carne enlatada Zenú & 8 & E452 & 340 \\
\hline Hamburguesa Colanta & 13 & E452 & 660 \\
\hline Albondigón Colanta & 10 & E451 & 420 \\
\hline Morcilla Colanta & 8 & $\mathrm{E} 452$ & 320 \\
\hline Nuggets de Pollo & 16 & $\mathrm{E} 439$ - E450 - E440 & 680 \\
\hline Tocineta ahumada Berna & 4,5 & E452 & 160 \\
\hline Alas rellenas Bucanero & 5 & E441 & 230 \\
\hline Pescado en salsa de tomate & 16,7 & 360 & 500 \\
\hline
\end{tabular}




\begin{tabular}{|c|c|c|c|}
\hline Cereales & & & \\
\hline Harina de maíz Maizena & 8 & E340 & 110 \\
\hline Harina de maíz postre Maizena & 5 & E341 & 60 \\
\hline Cereal de desayuno Nestlé & 2 & E340 & 90 \\
\hline Cereal de desayuno Tosh & 3 & E450 & 80 \\
\hline Cereal de desayuno Olímpica & 2 & E450 & 135 \\
\hline Frescavena Quaker & 1 & E340 & 20 \\
\hline \multicolumn{4}{|l|}{ Legumbres } \\
\hline Frijoles blancos Carulla & 0 & 250 & 0 \\
\hline Frijol rojo sabanero Carulla & 6 & 190 & 0 \\
\hline Frijol rojo Carulla & 6 & 129 & 0 \\
\hline Lentejas Carulla & 6,1 & 111 & 0 \\
\hline Garbanzo Carulla & 5,88 & 90 & 0 \\
\hline Arveja & 7 & 60 & 0 \\
\hline Arroz blanco Carulla & 8,6 & 70 & 0 \\
\hline Haba & 8,93 & 126 & 0 \\
\hline Maní & 9,9 & 8,6 & 0 \\
\hline Arroz blanco Diana & 5 & 75 & 10 \\
\hline Papa Fácil & 1 & 85 & 35 \\
\hline \multicolumn{4}{|l|}{ Chocolates } \\
\hline Cobertura de chocolate y leche & 0 & E341 & 60 \\
\hline Milo granulado & 3 & $375-\mathrm{E} 340$ & 75 \\
\hline Chocolisto Nestlé & 1 & $75-\mathrm{E} 340$ & 10 \\
\hline Café con leche Nescafé & 5 & E339 & 100 \\
\hline \multicolumn{4}{|l|}{ Panadería } \\
\hline Galletas tradicionales Saltín Noel & 6 & E339 & 88 \\
\hline Galletas Saltín Noel integral & 6 & E339 & 60 \\
\hline Galletas Tosh & 2 & E339 & 150 \\
\hline Galletas Nestlé Fitness & 2 & E339 & 230 \\
\hline Galletas Quaker con avena & 2 & E339 & 105 \\
\hline Galletas Club Social integral & 2 & E340 & 200 \\
\hline Galletas Club Social tradicional & 3 & E340 & 260 \\
\hline Galletas Milo & 2 & E340 & 110 \\
\hline Choco Barra & 2 & E340 & 60 \\
\hline Pan tajado Miel y Pasas Santa Clara & 4 & E341 & 160 \\
\hline \multicolumn{4}{|l|}{ Aderezos } \\
\hline Mayonesa Fruco & 0 & E338 & 115 \\
\hline Mayonesa Light & 0 & E338 & 125 \\
\hline Salsa rosada Fruco & 0 & E338 & 130 \\
\hline Sazonatodo Maggi & 0 & E341 & 0 \\
\hline Crema Maggi & 1 & E339 & 580 \\
\hline Crema de Leche Nestlé & 0 & E339 & 10 \\
\hline \multicolumn{4}{|l|}{ Gaseosas } \\
\hline Cocacola Zero & 0 & E338 & 60 \\
\hline Pespi Postobón & 0 & E338 & 60 \\
\hline Té frío Mercaldas & 0 & E3341 & 25 \\
\hline Gatorade & 0 & E340 & 110 \\
\hline Jugo de naranja Del Valle & 0 & E341 & 85 \\
\hline
\end{tabular}


La Administración de Alimentos y Medicamentos de los Estados Unidos (FDA por sus siglas en inglés: Food and Drugs Administration) define como aditivo "cualquier sustancia cuyo uso pretendido resulte o pueda razonablemente esperarse que resulte (directa o indirectamente) en que se convierta en un componente o afecte las características de cualquier alimento". Esta definición incluye cualquier sustancia utilizada en la producción, procesamiento, tratamiento, envasado, transporte o almacenamiento de alimentos y se divide en dos grupos. a) Aditivos alimentarios directos: aquellos que se añaden a un alimento para un propósito específico en ese alimento. Por ejemplo, la goma de xantano, utilizada en aderezos para ensaladas, leche con chocolate, rellenos de panadería, pudines y otros alimentos para añadir textura. La mayoría de los aditivos directos se identifican en la etiqueta entre los ingredientes de los alimentos. b) Aditivos alimentarios indirectos: aquellos que se convierten en parte del alimento en cantidades trazas, debido a su uso en embalaje, almacenamiento $u$ otro tipo de manipulación. Los fabricantes de envases de alimentos deben demostrar a la Administración de Alimentos y Medicamentos de los Estados Unidos (FDA) que todos los materiales que entran en contacto con los alimentos son seguros antes de que se les permita utilizarlos para tal fin.

Por otro lado, para la FDA un aditivo de color es cualquier colorante, pigmento o sustancia que, cuando se añade o se aplica a un alimento, fármaco o cosmético, o al cuerpo humano, es capaz (solo o mediante reacciones con otras sustancias) de impartir color. La FDA es responsable de regular todos los aditivos de color para asegurar que los alimentos que contienen aditivos de color sean seguros para comer, contengan sólo ingredientes aprobados y se etiqueten con precisión. ((http://www.fda.gov/ Food/IngredientsPackagingLabeling/FoodAdditivesIngredients/ucm094211.htm).
La FDA, sin embargo, no obliga a reportar en el etiquetado la cantidad de fósforo en sus diversas formas por lo que se incluye en la lista de ingredientes con nombres como fosfato monocalcico y fosfato de amonio.

En la normativa colombiana, en la Resolución 333 de 2011 (10 de febrero de 2011) del Ministerio de la Proteccion Social, "por la cual se establece el reglamento técnico sobre los requisitos de rotulado o etiquetado nutricional que deben cumplir los alimentos envasados para consumo humano", se encuentra referencia al fósforo en el capítulo III "Declaración de nutrientes", articulo 8 "Declaración y forma de presentación de los nutrientes", apartado 8.2 "Nutrientes de declaración opcional", 8.2.4: "vitaminas y minerales diferentes de la vitamina A, vitamina $\mathrm{C}$, hierro y calcio presentes naturalmente en el alimento se pueden declarar únicamente cuando se han establecido valores de referencia en el capítulo IV de este reglamento y se hallen en cantidades iguales o superiores al $2 \%$ del valor de referencia por porción del alimento"39. En el capítulo VI, "Declaraciones de propiedades de salud", artículo 23 "Declaraciones de propiedades de salud relacionadas con reducción de riesgos de enfermedad", apartado 23.1 "Calcio y osteoporosis": "el alimento debe cubrir o exceder los requisitos exigidos para el término o descriptor "alto" en calcio, conforme se define en la presente resolución; estar presente en una forma asimilable y el contenido de fósforo no puede ser superior al de calcio". No se encuentran a lo largo de la resolución referencias a otros aspectos relacionados con el fósforo.

Entre las posibles razones por las que no se reporta el contenido de fósforo total en dichos alimentos, hay que considerar que para esta medición los alimentos deberían ser sometidos a un análisis químico riguroso, procedimiento considerado poco práctico y costoso. 
En un estudio reciente se buscó correlacionar la ingesta de fósforo proveniente de fuentes orgánicas e inorgánicas con los niveles séricos, tanto en población sana como renal. Se encontró que compromiso de la función renal y bajo peso se correlacionaron con un efecto positivo significativo en los niveles de fósforo. Los alimentos que más impacto tuvieron fueron los lácteos, cereales y granos con fosfatos inorgánicos añadidos ${ }^{40}$. Estudios como los anteriores evidencian un mayor impacto de los alimentos con aditivos de fósforo, que contribuye en todas las implicaciones y en un peor control del enfermo renal. No cabe duda que estrategias de educación en este grupo de pacientes tendrán cabida en los resultados terapéuticos, tal como lo evidenció un estudio en pacientes con enfermedad renal en estadios avanzados a los que se controló el consumo de aditivos alimentarios ricos en fósforo. Efectivamente, se lograron significativas mejoras en los niveles de fósforo sérico (hasta $0,6 \mathrm{mg} / \mathrm{dl})^{41}$.

Otro grupo de estudios cuestiona las implicaciones de dietas bajas en fosfatos y, por lo tanto, en proteínas que podrían afectar el estado nutricional de los pacientes, generando así un impacto importante en el estado de salud global ${ }^{42,43}$. Una explicacion para dichos hallazgos puede encontrarse en los mecanismos, ya explicados en la presente revisión, que reconocen la limitación de los niveles séricos de fosfatos especialmente en estadios tempranos de la enfermedad. De ahí la necesidad de buscar en nuevos marcadores, una mejor correlación para un control más real de estos pacientes.

\section{Estrategias de manejo para control del fósforo}

Debemos reconocer que no está bajo el completo control de los pacientes la estimación del consumo diario de fósforo. Son amplias las limitaciones y comienzan por la dificultad en la identificación del contenido de este elemento en los alimentos más ampliamente consumidos y considerados de primera línea en la dieta promedio colombiana. Por ello, las estrategias de manejo al respecto deben estar encaminadas a ofrecer a nuestros pacientes recomendaciones sencillas, constantemente reforzadas por el grupo multidisciplinario a cargo, con el fin de crear cambios en los hábitos alimenticios que se mantengan en el tiempo y se adapten a la cultura y posibilidades económicas de nuestros pacientes.

Siguiendo estos propósitos, nuestro grupo de trabajo propone la implementación de una nueva herramienta educativa, adaptada para Colombia a partir de la iniciativa de un grupo de investigadores italianos publicada en el 2015. Ellos proponen una "Pirámide del fósforo" para instruir a los pacientes, de una forma sencilla, acerca de los alimentos no permitidos por su alto contenido en fosfatos, así como de aquellos adecuados para para su estado de salud $^{44}$ (Figura 2).

La pirámide consta de siete plantas, en las que los alimentos se disponen de acuerdo con el contenido de fósforo, la relación fósforo/proteína y la biodisponibilidad de fósforo de acuerdo con el grupo de alimentos. La distribución de alimentos en los distintos pisos apoya la elección, sin necesidad de memorizar el contenido de fósforo de cada alimento. Cada nivel tiene un color (de verde a rojo) según la frecuencia de ingesta recomendada, la cual varía entre "sin restricciones" y "evitar tanto como sea posible". En la base de la pirámide, el primer nivel (borde verde) contiene los alimentos con muy bajo contenido de fósforo (azúcar, aceite de oliva, alimentos libres de proteínas) o muy bajo en fósforo biodisponible (frutas y verduras). También incluye la clara de huevo que tiene un fósforo extremadamente favorable a la proporción de proteínas, al ser una fuente de proteínas de alto valor biológico y sin colesterol. 


\section{CONTENIDO DEL FÓSFORO EN LOS ALIMENTOS}

Los alimentos se distribuyen en siete niveles sobre la base de su contenido de fosforo, cada nivel, representado en color, de rojo a verde, pasando por naranja y amarillo que corresponde a la frecuencia de consumo recomendada, puede ser más alta para los alimentos de la base (verde con toma no restringida) y más baja para los alimentos de la punta (rojo, evitar en lo posibles).

- Los alimentos con fosforo desfavorable, relación de proteínas $>12 \mathrm{mg} / \mathrm{g}$ (Rojo a Naranja)

- Los alimentos con fosforo favorable, relación de proteínas $<12 \mathrm{mg} / \mathrm{g}$ (Verde a Naranja)

- Frutas y verduras deben utilizarse con precaución en pacientes en diálisis evitando la carga excesiva de potasio.

- Las grasas deben limitarse en pacientes con sobrepeso y obesidad. Evitar el consumo de alimentos energéticos.

- El azúcar debe evitarse en pacientes con diabetes y obesidad.

- Los productos libres de proteínas están recomendados a pacientes que no están en terapia de diálisis y que necesitan restricción de proteínas pero un consumo alto de energía.
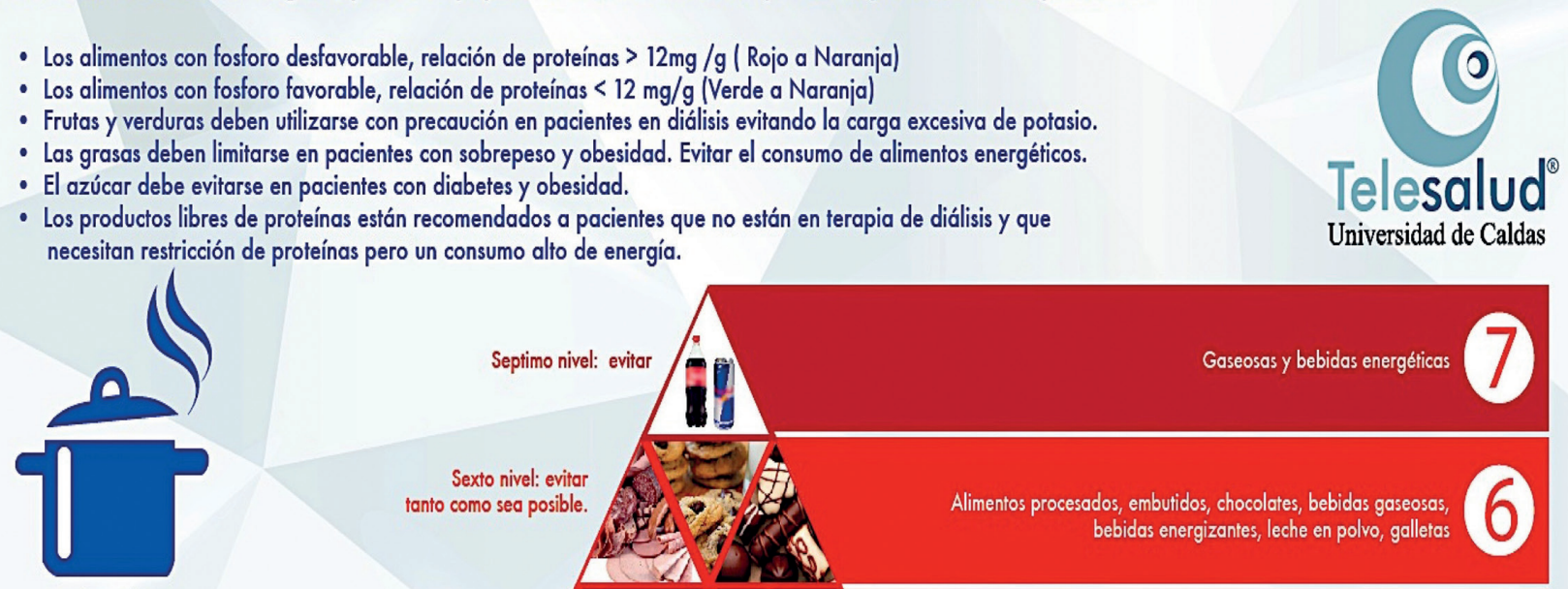

Hervir los alimentos y luego desechar el agua.

Después de hervidos pueden ser oliva o especias para mejorar ss sobor.

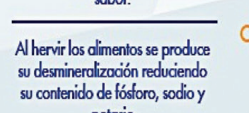
su contenido de fósforo, sodioy potasio
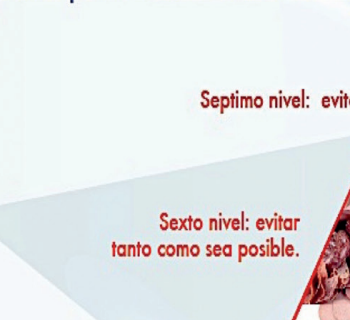

Universidad de Caldas

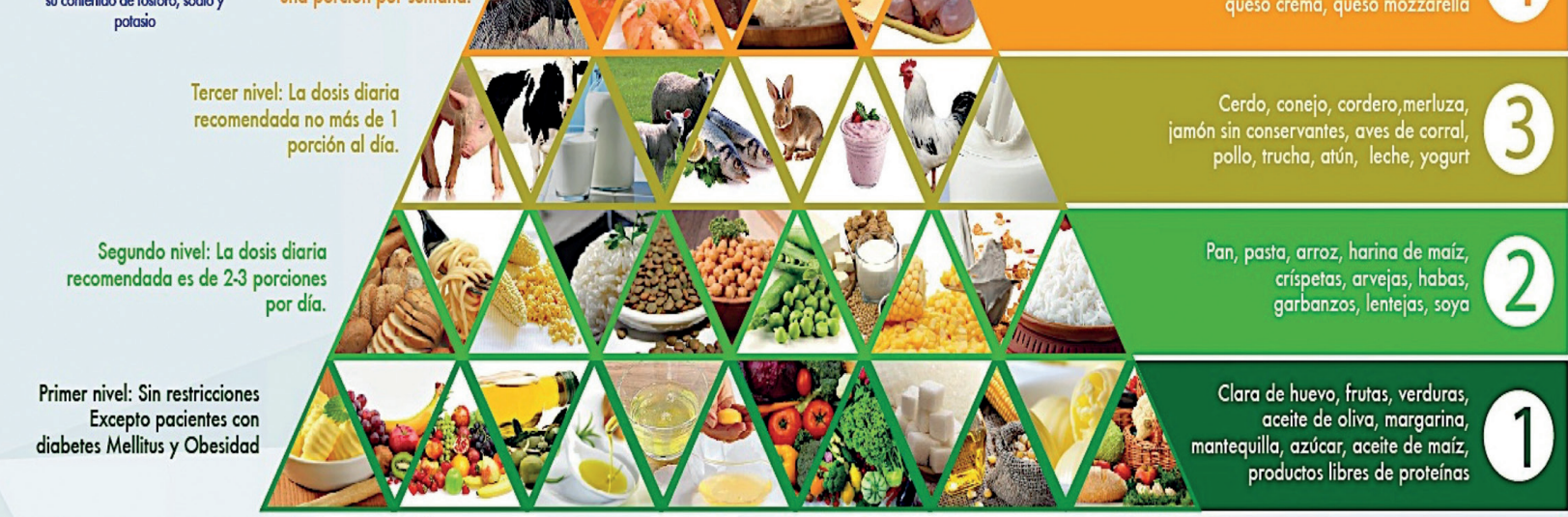

PIDA TODA LA INFORMACIÓN A SU NUTRICIONISTA

Fuente: traducida y adaptada al contexto Colombiano por Cesar Augusto Restrepo Valencia " D'Alessandro C, Piccoli GB A. The"phosphorus pyramyd": a visual tool for dietary phosphate management in dialiysis and CKD patients.BMC Nephrology 2015, 16:9.

Figura 2. Pirámide de contenido de fósforo en los alimentos. Tomado y adaptado de la referencia 44 (BMC Nephrol. 2015 Jan 20;16:9). 
La ingesta de estos alimentos es sin restricciones. Sin embargo, durante el asesoramiento, el profesional en salud debe tener consideraciones y advertencias especiales:

- Pacientes con diabetes, que deben evitar el azúcar y no exceder el consumo de frutas.

-Pacientes con sobrepeso u obesidad, deben reducir el azúcar, aceite de oliva, grasas vegetales y el consumo de mantequilla.

- Pacientes en diálisis deben limitar el consumo de frutas y verduras para evitar la ingesta excesiva de potasio.

-Por último, el uso de productos libres de proteínas está dirigido a pacientes con ERC sin terapia dialitica, quienes necesitan la restricción de proteínas, pero un alto consumo de energía.

El segundo nivel incluye principalmente alimentos vegetales, ricos en fósforo, pero principalmente como fitato, por lo tanto, con menor absorción intestinal. La dosis diaria recomendada es de 2 a 3 porciones por día. El tercer nivel incluye alimentos de origen animal y leche y sus derivados, con baja relación fosfato/proteina. La dosis diaria recomendada es de no más de 1 porción al día. El cuarto nivel muestra los alimentos con contenido de fósforo superiores a la proporción de proteína. La dosis diaria recomendada es de una porción por semana. El quinto nivel contiene los alimentos con alto contenido de fósforo. La dosis recomendada no más de 2 a 3 porción por mes. Finalmente, en la parte superior de la pirámide, el sexto y septimo nivel, incluye alimentos con aditivos con alto contenido de fósforo que se deben evitar tanto como sea posible.

La imagen de la olla hirviendo en el lado izquierdo sugiere la ebullición como el mejor método de cocción para reducir el contenido de fósforo. Este proceso provoca la desmineralización de los alimentos, reduciendo así el contenido de algunos minerales, como fósforo, sodio, potasio y calcio, tanto en vegetales como en productos derivados de animales. El grado de pérdida de mineral es proporcional a la cantidad de agua hirviendo que se utiliza, tamaño de las piezas, tiempo de cocción y ausencia de la cáscara para las plantas. Después de la ebullición, se ha reportado una reducción de fósforo del $51 \%$ para las verduras, $48 \%$ para las legumbres, y del $38 \%$ para la carne. Es oportuno destacar que la ebullición reduce el contenido de fósforo con una pérdida insignificante de nitrógeno, lo que lleva a un relación de fósforo más favorable a la proporción de proteína ${ }^{45,46}$.

\section{Conclusiones}

El reto al que nos enfrentamos es grande y sujeto a la influencia de nueva evidencia científica. Tenemos una clara asociación de los efectos deletéreos del fósforo en la salud de los pacientes con enfermedad renal, causa para que algunos autores lo llamen el "enemigo oculto", debido a su presencia desapercibida en los alimentos de consumo diario y por la gran limitación para el cumplimiento de las estrategias de control propuestas por los profesionales a cargo. Se requiere, por lo tanto, una educación ingeniosa y renovada ${ }^{47}$ acerca de la restricción dietaria de fosfatos en todos los pacientes, incluso desde estadios tempranos de la enfermedad, pues es la única alternativa con la que contamos para impactar en el pronóstico de dichos pacientes, antes de que sea manifiesta la hiperfosfatemia y necesitemos, como alternativa, de una terapia farmacológica ${ }^{48-50}$.

\section{Agradecimientos}

A todo el equipo de Telesalud de la Universidad de Caldas, quienes nos colaboraron en la elaboración de las gráficas.

\section{Responsabilidades éticas}

Protección de personas y animales

Los autores declaran que para esta investigación no se han realizado experimentos en seres humanos ni en animales. 


\section{Confidencialidad de los datos}

Los autores declaran que en este artículo no aparecen datos de pacientes.

\section{Derecho a la privacidad y consentimiento} informado

Los autores declaran que en este artículo no aparecen datos de pacientes.

\section{Conflicto de interés}

Los autores declaran no tener conflicto de interés.

\section{Financiación:}

No se contó con fuentes de financiación. 


\section{Referencias}

1. Martinez I, Saracho R. El fósforo, ¿héroe o villano?. NefroPlus (2009);2:1-8.

2. Johnson RJ, Feehally J, Floege Jurgen. Comprehensive Clinical Nephrology. 5th ed. Amsterdam: Elsevier Saunders; 2015. Chapter 10, Disorders of Calcium, Phosphate, and Magnesium Metabolism; p. 132

3. Penido MG, Alon US. Phosphate homeostasis and its role in bone health. Pediatr Nephrol [Internet]. 2012 [accedido 01 Jun 2000];27(11):2039-48. Available from: https://doi.org/10.1007/s00467-012$2175-\mathrm{z}$

4. Blaine J, Chonchol M, Levi M. Renal Control of Calcium, Phosphate, and Magnesium Homeostasis. Clin J Am Soc Nephrol [Internet]. 2015 [accedido 01 Jun 2000];10(7):1257-72. Available from: https://doi.org/10.2215/CJN.09750913 .

5. Kestenbaum B. Phosphate Metabolism in the Setting of Chronic Kidney Disease: Significance and Recommendations for Treatment. Sem Dial [Internet]. 2007 [accedido 01 Jan 2000];20(4):286-94. Available from: https://doi.org/10.1111/j.1525-139X.2007.00303.

6. Blaine J, Weinman EJ, Cunningham R. The regulation of renal phosphate transport. Adv Chronic Kidney Dis [Internet]. 2011 [accedido 01 Jan 2000];18(2):77-84. Available from: https://doi.org/10.1053/j. ackd.2011.01.005.

7. Biber J, Hernando N, Forster I, Murer H. Regulation of phosphate transport in proximal tubules. Pflugers Arch [Internet]. 2009 [accedido 01 Jan 2000];458(1):39-52. Available from: https://doi. org/10.1007/s00424-008-0580-8

8. Dusso AS, Brown AJ, Slatopolsky E. Vitamin D. Am J Physiol Renal -Physiol [Internet]. 2005 [accedido 01 Jan 2000];289(1):F8-F28. Available from: https://doi.org/10.1152/ajprenal.00336.2004

9. Komaba H, Kakuta T, Fukagawa M. Disease of the parathyroid gland in chronic kidney disease. Clin Exp Nephrol [Internet]. 2011 [accedido 01 Jan 2000];15(6):797-809. Available from: https://doi. org/10.1007/s10157-011-0502-5

10. Restrepo CA. Nefrología Básica. 2da ed. Manizales. Editorial La Patria; 2012. Capítulo 26; p. 251: Desordenes óseos y minerales en la enfermedad renal crónica.

11. Block GA, Klassen PS, Lazarus JM, Ofsthun N, Lowrie EG, Chertow GM. Mineral metabolism, mortality, and morbidity in maintenance hemodialysis. J Am Soc Nephrol [Internet]. 2004 [accedido 01 Jan 2000];15(8):2208-18. Available from: https://doi.org/10.1097/01.ASN.0000133041.27682.A2

12. Huang CL, Moe OW. Clinical assessment of phosphorus status, balance and renal handling in normal individuals and in patients with chronic kidney disease. Curr Opin Nephrol Hypertens [Internet]. 2013 [accedido 01 Jan 2000];22(4):452-8. Available from: https://doi.org/10.1097/MNH. 0b013e328362483a

13. Ori Y, Herman M, Tobar A, Chernin G, Gafter U, Chagnac A, Izhak OB, Korzets A. Acute phosphate nephropathy: An emerging threat. Am J Med Sci [Internet]. 2008 [accedido 01 Jan 2000];336(4):30914. Available from: https://doi.org/10.1097/MAJ.0b013e318167410c

14. Markowitz GS, Nasr SH, Klein P, Anderson H, Stack JI, Alterman L, Price B, Radhakrishnan J, D'Agati VD. Renal failure due to acute nephrocalcinosis following oral sodium phosphate bowel cleansing. Hum Pathol [Internet]. 2004 [accedido 01 Jan 2000];35(6):675-84. Available from: https:// doi.org/10.1016/j.humpath.2003.12.005 
15. Lien YH. Is bowel preparation before colonoscopy a risky business for the kidney?. Nat Clin Pract Nephrol [Internet]. 2008 [accedido 01 Jan 2000];4(11):606-14. Available from: https://doi.org/10.1038/ ncpneph0939

16. Caravaca F, Villa J, Garcia de Vinuesa E, Martinez del Viejo C, Martinez Gallardo R, Macias R, Ferreira F, Cerezo I, Hernandez-Gallego R. Relationship between serum phosphorus and the progression of advanced chronic kidney disease. Nefrología [Internet]. 2011 [accedido 01 Jan 2000];31(6):707-15. Available from: https://doi.org/10.3265/Nefrologia.pre2011.Sep.11089.

17. Voormolen N, Noordzij M, Grootendorst DC, Beetz I, Sijpkens YW, van Manen JG, et al. High plasma phosphate as a risk factor for decline in renal function and mortality in pre-dialysis patients. Nephrol Dial Transplant [Internet]. 2007 [accedido 01 Jan 2000];22(10):2909-16 Available from: https://doi. org/10.1093/ndt/gfm286

18. Chue CD, Edwards NC, Davis LJ, Steeds RP, Townend JN, Ferro CJ. Serum phosphate but not pulse wave velocity predicts decline in renal function in patients with early chronic kidney disease. Nephrol Dial Transplant [Internet]. 2011 [accedido 01 Jan 2000];26(8):2576-82Available from: https://doi. org/10.1093/ndt/gfq787

19. Kuzela DC, Huffer WE, Conger JD, Winter SD, Hammond WS. Soft tissue calcification in chronic dialysis patients. Am J Pathol [Internet]. 1977 [accedido 01 Jan 2000];86(2):403-24. Available from: https://www.ncbi.nlm.nih.gov/pmc/articles/PMC2032079/

20. Tonelli M, Sacks F, Pfeffer M, Gao Z, Curhan G; Cholesterol and Recurrent Events Trial Investigators. Relation between serum phosphate level and cardiovascular event rate in people with coronary disease. Circulation [Internet]. 2005 [accedido 01 Jan 2000];112(17):2627-33. Available from: https:// doi.org/10.1161/CIRCULATIONAHA.105.553198

21. Foley RN, Collins AJ, Herzog CA, Ishani A, Kalra PA. Serum Phosphorus Levels Associate with Coronary Atherosclerosis in Young Adults. J Am Soc Nephrol [Internet]. 2009 [accedido 01 Jan 2000];20(2):397-404. Available from: https://doi.org/10.1681/ASN.2008020141

22. Murtaugh MA, Filipowicz R, Baird BC, Wei G, Greene T, Beddhu S. Dietary phosphorus intake and mortality in moderate chronic kidney disease: NHANES III. Nephrol Dial Tarnsplant [Internet]. 2012 [accedido 01 Jan 2000];27(3):990-6. Available from: https://doi.org/10.1093/ndt/gfr367

23. Takeda E, Yamamoto H, Yamanaka-Okumura H, Taketani Y. Dietary phosphorus in bone health and quality of life. Nutr Rev [Internet]. 2012 [accedido 01 Jan 2000];70(6):311-21. Available from: https:// doi.org/10.1111/j.1753-4887.2012.00473.x

24. Adeney KL, Siscovick DS, Ix JH, Seliger SL, Shlipak MG, Jenny NS, Kestenbaum BR. Association of serum phosphate with vascular and valvular calcification in moderate CKD. J Am Soc Nephrol [Internet]. 2009 [accedido 01 Jan 2000];20(2):381-7. Available from: https://doi.org/10.1681/ ASN.2008040349

25. Chue CD, Edwards NC, Moody WE, Steeds RP, Townend JN, Ferro CJ. Serum phosphate is associated with left ventricular mass in patients with chronic kidney disease: a cardiac magnetic resonance study. Heart [Internet]. 2012 [accedido 01 Jan 2000];98(3):219-24. Available from: https://doi.org/10.1136/ heartjnl-2011-300570

26. Yamamoto KT, Robinson-Cohen C, de Oliveira MC, Kostina A, Nettleton JA, Ix JH, Nguyen H, Eng J, Lima JA, Siscovick DS, Weiss NS, Kestenbaum B. Dietary phosphorus is associated with greater left ventricular mass. Kidney Int [Internet]. 2013 [accedido 01 Jan 2000];83(4):707-14. Available from: https://doi.org/10.1038/ki.2012.303 
27. Kestenbaum B, Sampson JN, Rudser KD, Patterson DJ, Seliger SL, Young B, Sherrard DJ, Andress DL. Serum Phosphate Levels and Mortality Risk among People with Chronic Kidney Disease. J Am Soc Nephrol [Internet] 2005 [accedido 01 Jan 2000];16(2):520-58. Available from: https://doi. org/10.1681/ASN.2004070602

28. Vervloet MG, van Ittersum FJ, Buttler RM, Heijboer AC, Blankenstein MA, ter Wee PM. Effects of dietary phosphate and calcium intake on fibroblast growth factor-23. Clin J Am Soc Nephrol [Internet] 2011 [accedido 01 Jan 2000];6(2):383-9. Available from: https://doi.org/10.2215/CJN.04730510

29. Kuro-o M. A phosphate-centric paradigm for pathophysiology and therapy of chronic kidney disease. Kidney Int Suppl [Internet]. 2013 [accedido 01 Jan 2000];3(5),420-6. Available from: http://dx.doi. org/10.1038/kisup.2013.88

30. Goto S, Nakai K, Kono K, Yonekura Y, Ito J, Fujii H, Nishi S. Dietary phosphorus restriction by a standard low-protein diet decreased serum fibroblast growth factor 23 levels in patients with early and advanced stage chronic kidney disease. Clin Exp Nephrol [Internet]. 2014 [accedido 01 Jan 2000];18(6):925-31. Available from: https://doi.org/10.1007/s10157-014-0947-4

31. Mehrotra R, Peralta CA, Chen SC, Li S, Sachs M, Shah A, Saab G, Whaley-Connell A, Kestenbaum B, McCullough PA; Kidney Early Evaluation Program Investigators. No independent association of serum phosphorus with risk for death or progression to end-stage renal disease in a large screen for chronic kidney disease. Kidney Int [Internet]. 2013 [accedido 01 Jan 2000];84(5):989-97. Available from: https://doi.org/10.1038/ki.2013.145

32. Noori N, Sims JJ; Kopple JD, Shah A, Colman S, Shinaberger CS, Bross R, Mehrotra R, Kovesdy C, Kalantar-Zadeh K. Organic and inorganic dietary phosphorus and its management in chronic kidney disease. Iran J Kidney Dis [Internet]. 2010 [accedido 01 Jan 2000];4(2):89-100. Available from: http:// www.ijkd.org/index.php/ijkd/article/view/345/176

33. Kalantar-Zadeh K, Gutekunst L, Mehrotra R, Kovesdy CP, Bross R, Shinaberger CS, Noori N, Hirschberg R, Benner D, Nissenson AR, Kopple JD. Understanding sources of dietary phosphorus in the treatment of patients with chronic kidney disease. Clin J Am Soc Nephrol [Internet]. 2010 [accedido 01 Jan 2000];5(3):519-30. Available from: https://doi.org/10.2215/CJN.06080809

34. Lou-Arnal LM, Arnaudas-Casanova L, Caverni-Muñoz A, Vercet-Tormo A, Caramelo-Gutierrez R, Munguia-Navarro P, Campos-Gutierrez B, Garcia-Mena M, Moragrera B, Moreno-Lopez R, Bielsa-Gracia S, Cuberes-Izquierdo M; Grupo de Investigación ERC Aragón. Fuentes ocultas de fósforo: presencia de aditivos con contenido en fósforo en los alimentos procesados. Nefrología [Internet]. 2014 [accedido 01 Jan 2000];34(4):498-506. Available from: https://doi.org/10.3265/Nefrologia. pre2014.Apr.12406

35. Moe SM, Zidehsarai MP, Chambers MA, Jackman LA, Radcliff JS, Trevino LL, Donahue SE, Asplin JR. Vegetarian compared with meat dietary protein source and phosphorus homeostasis in chronic kidney disease. Clin J Am Soc Nephrol [Internet]. 2011 [accedido 01 Jan 2000];6(2):257-64. Available from: https://doi.org/10.2215/CJN.05040610

36. Fukagawa M, Komaba H, Miyamoto K. Source matters: from phosphorus load to bioavailability. Clin J Am Soc Nephrol [Internet]. 2011 [accedido 01 Jan 2000];6(2):239-40. Available from: https://doi. org/10.2215/CJN.11051210

37. Calvo M S, Uribarri J. Contributions to total phosphorus intake: All sources considered. Semin Dial [Internet]. 2013 [accedido 01 Jan 2000];26(1):54-61. Available from: https://doi.org/10.1111/ sdi. 12042

38. REGLAMENTO (UE) No 1129/2011 DE LA COMISIÓN de 11 de noviembre de 2011 por el que se 
modifica el anexo II del Reglamento (CE) no 1333/2008 del Parlamento Europeo y del Consejo para establecer una lista de aditivos alimentarios de la Unión.

39. Resolución 333 de 2011 Por la cual se establece el reglamento técnico sobre los requisitos de rotulado o etiquetado nutricional que deben cumplir los alimentos envasados para consumo humano. (Ministerio de la Protección Social 10 de Febrero de 2011)

40. Moore LW, Nolte JV, Gaber AO, Suki WN. Association of dietary phosphate and serum phosphorus concentration by levels of kidney function. Am J Clin Nutr [Internet]. 2015 [accedido 01 Jan 2000];102(2):444-53. Available from: https://doi.org/10.3945/ajcn.114.102715

41. Sullivan C, Sayre SS, Leon JB, Machekano R, Love TE, Porter D, Marbury M, Sehgal AR. Effect of Food Additives on Hyperphosphatemia Among Patients With End-stage Renal Disease A Randomized Controlled Trial. JAMA [Internet]. 2009 [accedido 01 Jan 2000];301(6):629-35. Available from: https://doi.org/10.1001/jama.2009.96

42. Selamet U, Tighiouart H, Sarnak MJ, Beck G, Levey AS, Block G, Ix JH. Relationship of dietary phosphate intake with risk of end-stage renal disease and mortality in chronic kidney disease stages 3-5: The Modification of Diet in Renal Disease Study. Kidney Int [Internet]. 2016 [accedido 01 Jan 2000];89(1):176-84. Available from: https://doi.org/10.1038/ki.2015.284

43. Shinaberger CS, Greenland S, Kopple JD, Van Wyck D, Mehrotra R, Kovesdy CP, Kalantar-Zadeh K. Is controlling phosphorus by decreasing dietary protein intake beneficial or harmful in persons with chronic kidney disease?. Am J Clin Nutr [Internet]. 2008 [accedido 01 Jan 2000];88(6):1511-18. Available from: https://doi.org/10.3945/ajcn.2008.26665

44. D’Alessandro C, Piccoli GB, Cupisti A. The "phosphorus pyramid": a visual tool for dietary phosphate management in dialysis and CKD patients. BMC Nephrol [Internet]. 2015 [accedido 01 Jan 2000];16:9. Available from: https://doi.org/10.1186/1471-2369-16-9

45. Cupisti A, Comar F, Benini O, Lupetti S, D'Alessandro C, Barsotti G, Gianfaldoni D. Effect of boiling on dietary phosphate and nitrogen intake. J Renal Nutr [Internet]. 2006 [accedido 01 Jan 2000];16(1):36-40. Available from: https://doi.org/10.1053/j.jrn.2005.10.005

46. Jones WL. Demineralization of a wide variety of foods for the renal patient. J Renal Nutr [Internet]. 2001 [accedido 01 Jan 2000];11(2):90-6. Available from: https://doi.org/10.1016/S1051-2276(01)38751-4

47. Reddy V, Symes F, Sethi N, Scally AJ, Scott J, Mumtaz R, Stoves J. Dietitian-Led education program to improve phosphate control in a single-center hemodialysis population. J Renal Nutr [Internet]. 2009 [accedido 01 Jan 2000];19(4):314-20. Available from: https://doi.org/10.1053/j.jrn.2008.11.001

48. Gonzales-Parra E, Gracia-Iguacel C, Egido J, Ortiz A. Phosphorus and nutrition in chronic kidney disease. Int J Nephrol [Internet]. 2012 [accedido 01 Jan 2000];2012:[5 p.]. Available from: https://doi. org/10.1155/2012/597605

49. Kalantar-Zadeh K. Patient education for phosphorus management in chronic kidney disease. Patient Prefer Adherence [Internet]. 2013 [accedido 01 Jan 2000];7:379-90. Available from: https://doi. org/10.2147/PPA.S43486

50. Tonelli M. Serum Phosphorus in people with chronic kidney disease: you are what you eat. Kidney Int [Internet]. 2013 [accedido 01 Jan 2000];84(5):871-3. Available from: https://doi.org/10.1038/ ki.2013.258 